\begin{tabular}{|c|c|c|c|}
\hline Eiszeitalter u. Gegenwart & $\mathbf{3 0}$ & $\begin{array}{c}109-123 \\
\mathrm{Abb} ., 2 \mathrm{Taf} .\end{array}$ & Hannover 1980 \\
\hline
\end{tabular}

\title{
Neuester Stand der Quartärforschung in Neuguinea
}

\author{
ERNST LÖFFLER *)
} Massif, glaciation, gradient, temperature depression, glacier, deglaciation, pollen, C-14 dating,
tropical zone, Pleistocene, New Guinea

Kurzfassung: Die eiszeitlichen Klimaschwankungen führten auch auf Neuguinea zu einer beachtlichen Vergletscherung der Hochgebirge, und rund $2000 \mathrm{~km}^{2}$ waren von Eis bedeckt. Die eiszeitliche Schneegrenze lag bei ungefähr $3550 \mathrm{~m}$, aber es gab einige Schwankungen aufgrund lokaler klimatischer Verhältnisse oder in einem Fall wegen tektonischer Ereignisse. Der älteste Nachweis für das Vorhandensein eines Gletschers ist ein Palagonit, der etwa 700000 Jahre alt ist, und der entstand, als am Mt. Giluwe eine vulkanische Eruption von Lava unter Eis stattfand. Die Datierung ist allerdings mit einem großen Unsicherheitsfaktor verbunden. Eine weitere Episode vulkanischer Aktivität und Gletscherbildung konnte für die Zeit um 300000 a.B.P. nachgewiesen werden.

Die letzte Vereisung war fast überall die ausgedehnteste und ihr Maximum wurde zwischen 18500 und 16000 a B.P. erreicht, sie entspricht also der Würm-Vereisung. Ab etwa 15000 a B.P. setzte der Gletscherrückzug ein, und um 10000-9000 a B.P. waren die Gletscher verschwunden, wahrscheinlich auch dort, wo heute wieder kleinere Gletscher vorhanden sind. Diese entstanden nach 5000 a B.P.

Die Rekonstruktion des letzteiszeitlichen Klimas ist mit Schwierigkeiten verbunden, da glazialmorphologische und pollenanalytische Befunde unterschiedliche Depressionen der Höhenstufen und Temperaturen anzeigen. Der Schneegrenzdepression von $1000 \mathrm{~m}$ steht eine Depression der Waldgrenze von $1500 \mathrm{~m}$ oder mehr gegenüber und die entsprechenden Temperaturdepressionen sind $5,5^{\circ} \mathrm{C}$ und $10^{\circ} \mathrm{C}$. Es wird argumentiert, daß die im Pollenbild nachgewiesene starke Depression der Höhenstufen möglicherweise lokale Bedingungen reflektiert und nicht eine allgemeine Temperaturabsenkung von $10^{\circ} \mathrm{C}$.

Die eiszeitliche Temperaturdepression hatte erstaunlich geringe Auswirkungen auf andere geomorphologische Prozesse wie Solifluktion und fluvioglaziale Vorgänge. Dies wird auf die extreme Einförmigkeit des tropischen Tageszeitenklimas zurückgeführt, das nur einen schmalen Höhensaum regelmäßiger Nachtfröste zuläßt und das Schmelzregime der Gletscher einem relativ regelmäßigen, tageszeitlichen und nicht jahreszeitlichen Zyclus unterwirft.

\section{[Present State of Knowledge on Quaternary Research in New Guinea]}

A b s t r a c t : The Pleistocene temperature depression resulted in a relatively extensive glacial ice cover in the high montains of New Guinea and approximately $2000 \mathrm{~km}^{2}$ were covered by ice. The Pleistocene snowline was at approximately $3550 \mathrm{~m}$ altitude and there were only minor variations due to local climatic conditions or in one case due to tectonic events. The oldest proof of glacial ice is a palagonite which may date back to about 700000 years and which formed when eruptions of lava took place under ice on Mt Giluwe. Due to atmospheric contamination of the argon, the date is however not very reliable. A further episode of volcanic activity and glaciation could be established with greater certainty for the time between about 290000 and 300000 years B.P. on the same mountain.

The maximum extent of the glaciation was reached in the last glaciation between about 18500 and 16000 years B.P. This corresponds to the Würm glaciation in the northern hemisphere. From 15000 B.P. onward the glaciers retreated and by about 10000-9000 years B.P. the glaciers had completely disappeared, even probably from the areas which are presently glaciated. These glaciers seem to have developed later from about 5000 years B.P. as a result of a slight drop in temperature. The reconstruction of the climate during the last glaciation has proved difficult because of conflicting results from geomorphological and palynological data. While the depression of the

*) Anschrift des Verfassers: Prof. Dr. E. L ö f $\mathrm{fle} r$, Geogr. Inst., Universität Mainz. Zur Zeit: 9 Lyndon St. Kaleen, ACT 2617, Australien. 
snowline was in the order of $1000 \mathrm{~m}$, the depression of the forest/grassland boundary seems to have been nearly twice that much. It is argued the the extraordinarily large depression in the forest boundary could be due to local climatic conditions and not to a general depression in temperatures by $10^{\circ} \mathrm{C}$. There is also no evidence for this large depression of the vegetation belts in the Lake Trist area.

The Pleistocene temperature depression had little effect on other geomorphological processes like periglacial solifluction and fluvioglacial activity. This is explained by the extreme uniformity of the tropical climate in New Guinea resulting in a very narrow belt of diurnal frosts and in a diurnal regime of melting of the glaciers resulting in an evenly spread discharge of meltwater throughout the year.

\section{Einführung}

Die späte Durchdringung und Exploration Neuguineas durch die Kolonialmächte bewirkte, daß trotz vielversprechender Ansätze zur Zeit der deutschen Kolonialherrschaft im nordöstlichen Teil der Insel eine eigentliche geowissenschaftliche Forschungstätigkeit nur etwa zwei Jahrzehnte zurückliegt, aber da man in vielen Gebieten sogleich mit modernen Forschungsmethoden arbeitete, wie Pollenanalyse, ${ }^{14} \mathrm{C}-, \mathrm{K}-\mathrm{Ar}$ - und ${ }^{230} \mathrm{Th} /{ }^{234} \mathrm{U}$ Datierungen, und Zugang zu Luftbildern hatte, kann man Neuguinea heute ohne Zweifel als eines der besser erforschten tropischen Gebiete bezeichnen. Dies gilt insbesondere für das Hochgebirge, dessen glazial überformte Landschaften zu den offensichtlichsten Zeugen quartärer Klimaschwankungen gehören.

Die Existenz rezenter Gletscher in den höchsten Gebirgsabschnitten Neuguineas ist schon seit langem bekannt, praktisch schon seit der ersten Sichtung der Gebirge durch den Holländer Carstensz, dessen Name der höchste Gebirgsabschnitt auch bis vor einem Jahrzehnt trug. Es dauerte allerdings bis 1912, bis der Gletscher erstmals von Europäern besucht wurde. Der Engländer WOLLASTON erreichte in diesem Jahr die Gletscherzunge nach mühsamem und gefährlichem Aufstieg (Wollaston 1914). Die nächste Expedition, die bis zu diesem Gletscher vordrang, war eine holländische Forschungsexpedition unter A. H. Colıjn, die erstmals Messungen und genauere Kartierungen durchführte. Außerdem wurde erstmals die Ausdehnung der pleistozänen Vereisung untersucht und Dozy, der Glazialgeologe der Expedition, behauptete, die Maximalausdehnung der eiszeitlichen Gletscher hätte Höhenlagen um 2000 m erreicht, eine erstaunlich tiefe Lage für Gletscher in der Nähe des Äquators (Dozy 1938).

Nach dem Weltkrieg wurde das Gletschergebiet von mehreren Expeditionen mit hauptsächlich bergsteigerischer Zielsetzung aufgesucht, aber Anfang der siebziger Jahre unternahm eine Gruppe von Wissenschaftlern aus verschiedenen australischen Universitäten zwei ausgedehntere Expeditionen in das Gebirge und führten eine Reihe ausgezeichneter Untersuchungen über den rezenten Gletscherhaushalt, die jüngsten Gletscherschwankungen, die Seen, Vegetation und das Klima durch (Hope et al. 1976). Das Hauptziel der Expeditionen war die Untersuchung der rezenten Verhältnisse, aber einige wichtige Beobachtungen über die pleistozänen Gletscherschwankungen wurden ebenfalls gemacht.

Im östlichen Neuguinea, das unter australischer Verwaltung bzw. bis 1918 zum Teil unter deutscher Verwaltung stand, setzte eine intensivere Forschung in den sechziger Jahren ein. Es gab allerdings einen kurzen Bericht über das Vorkommen eiszeitlicher Gletscherspuren im Saruwaged-Gebirge von Detzner (1933), dessen Name jedoch weniger mit dieser Entdeckung verbunden ist, als vielmehr mit der Tatsache, daß er während des ersten Weltkriegs im Innern Neuguineas überlebte und anschließend über seine Erlebnisse ein Buch mit dem Titel „Vier Jahre unter Kannibalen“ schrieb. Dieses Buch zog nach nachweisbar falschen Behauptungen viel Kritik auf sich und DETZNERs tatsächlich korrekten Beobachtungen wurde daher auch kein Glaube geschenkt. 
Eine erste wichtige Veröffentlichung über die Vergletscherung des Mt Wilhelm-Gebirges, des höchsten Gebirges Ostneuguineas, erschien 1960 (REINER 1960), dann folgten Ende der sechziger und im Laufe der siebziger Jahre eine ganze Reihe von Arbeiten zum Teil geomorphologischer zum Teil pollenanalytischer Natur, die wesentlich zur Erweiterung unseres Wissens über die quartäre Geschichte dieses Raumes beitrugen (HOPE 1976; Hope \& Peterson 1975; Löffler 1970, 1971, 1972, 1976; Walker 1970, 1979). Im folgenden möchte ich die wichtigsten Befunde zusammenfassen und die daraus resultierende Problematik diskutieren.

\section{Die Hauptvergletscherung}

Zeugen der letzten Vereisung sind auf allen Gebirgen zu finden, die rund $3600 \mathrm{~m}$ Höhenlage übersteigen. Die durch das Eis geschaffenen Oberflächenformen heben sich immer sehr deutlich von den tiefer liegenden fluviatil geprägten Kerbtallandschaften ab, und dies drückt sich besonders deutlich im Luftbild aus (Taf. I). Akzentuiert wird der Gegensatz von glazialen und nicht glazialen Tälern noch durch die Gegenwart von Grasländern in den breiten glazial überformten Talböden. Es handelt sich hierbei jedoch nicht um eine klimatische Erscheinung, sondern um eine vornehmlich edaphische, in dem die glaziale Erosion die topographischen Voraussetzungen für die relativ tief liegenden Grasländer geschaffen hat. Anthropogene Einflüsse vor allem durch den Gebrauch des Feuers haben die Grasländer zusätzlich noch stark erweitert (LöFfLER 1979 a).

Der glaziale Formenschatz der Gebirge ist ausgezeichnet erhalten und umfaßt mächtige Kare mit steilen Karrückwänden, Karschwellen, Rundhöcker, Stufen, Trogtäler und mächtige gut erhaltene Moränen. Die Moränenrücken sind mitunter sehr prominent und es erscheint zunächst erstaunlich, daß sich derartige leicht erodierbare Formen in dieser Gebirgslandschaft erhalten konnten. Der ausgezeichnete Erhaltungszustand von Moränen scheint jedoch ein typisches Kennzeichen tropischer Gebirgsvergletscherung zu sein und wurde auch aus dem tropischen Afrika und Südamerika beschrieben (DownIE \& WILKINson 1972; Tricart et al. 1962). Ein wichtiger Grund für den guten Erhaltungszustand dürfte im tropischen Tageszeitenklima liegen und damit in dem über das ganze Jahr verteilten Schmelzregime der Gletscher. Selbst beim Gletscherrückzug während einer allgemeinen Erwärmung gibt es keine Zeiten besonders starker Wasserführung und damit Erosion. Dies erklärt auch den Mangel an ausgedehnteren fluvioglazialen Ablagerungen.

\subsection{Mt. Giluwe}

Das interessanteste Gebirge Neuguineas vom Gesichtspunkt der Vergletscherung ist der Mt. Giluwe, ein mächtiger erloschener Vulkan, der mit knapp unter $4400 \mathrm{~m}$ Gipfelhöhe den zweithöchsten Gipfel Ostneuguineas darstellt (Abb. 1). Mit einer $180 \mathrm{~km}^{2}$ großen Gletscherkappe stellt er das mit Abstand ausgedehnteste Gletschergebiet Ostneuguineas dar. Die mehr oder weniger zusammenhängende Gletscherkappe überdeckte das gesamte Gipfelgebiet bis etwa $3200 \mathrm{~m}$ Höhe, und von dort zweigten sich einige kurze Talgletscher $a b$ und stießen einige hundert Meter tiefer vor. Die Endmoränen der Maximalausdehnung sind ausgezeichnet erhalten und umgeben den gesamten Vulkan wie ein Gürtel. Besonders auffallend sind die zahlreichen, niedrigen Rückzugsmoränen, die vor allem die relativ flachen und wenig zerschnittenen Ost- und Westhänge bedecken (Taf. II). Insgesamt sind etwa 20 einzelne Moränenrücken auszumachen. Ich habe diese in 5 Gruppen unterteilt, die wahrscheinlich die Hauptstadien des Rückzugs anzeigen (LöffLER 1972). Die tiefsten Rückzugsmoränen überlagern z. T. die mächtige und breite Moräne der Maximalvereisung, ein Hinweis darauf, daß es sich während dieses Rückzugs bereits um einen sehr gering mächtigen Gletscher gehandelt haben muß, der praktisch keine Erosionskraft 


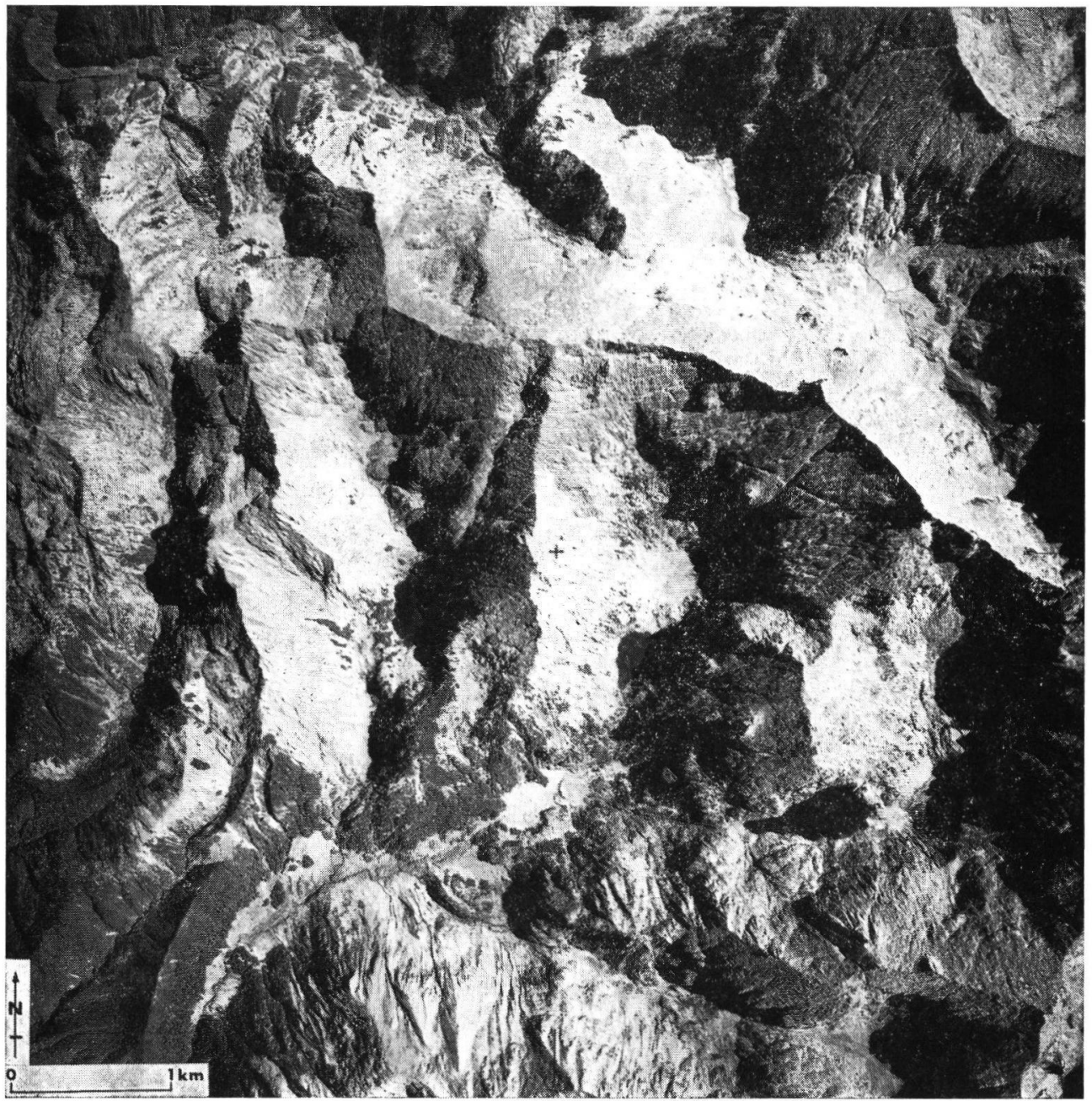

Taf. I: Mt Wilhelm mit tief eingesenkten Karen, steilen Karrückwänden, Karseen und mächtigen Endmoränen am Ausgang der Trogtäler (links unten). Eine gewisse Hangasymmetrie mit steilen westexponierten und weniger steilen ostexponierten Hängen ist auf dem Luftbild zu erkennen, und ist wahrscheinlich bedingt durch den etwas günstigeren Ansatz zur Gletscherbildung an westexponierten Hängen, die aufgrund der regelmäßigen Wolkenbildung um die Mittagszeit eine etwas geringe Einstrahlung erhalten als ostexponierte Hänge. Diese Unterschiede kommen jedoch nur zum Tragen, wenn es sich um stark zergliederte Gebirge handelt. 


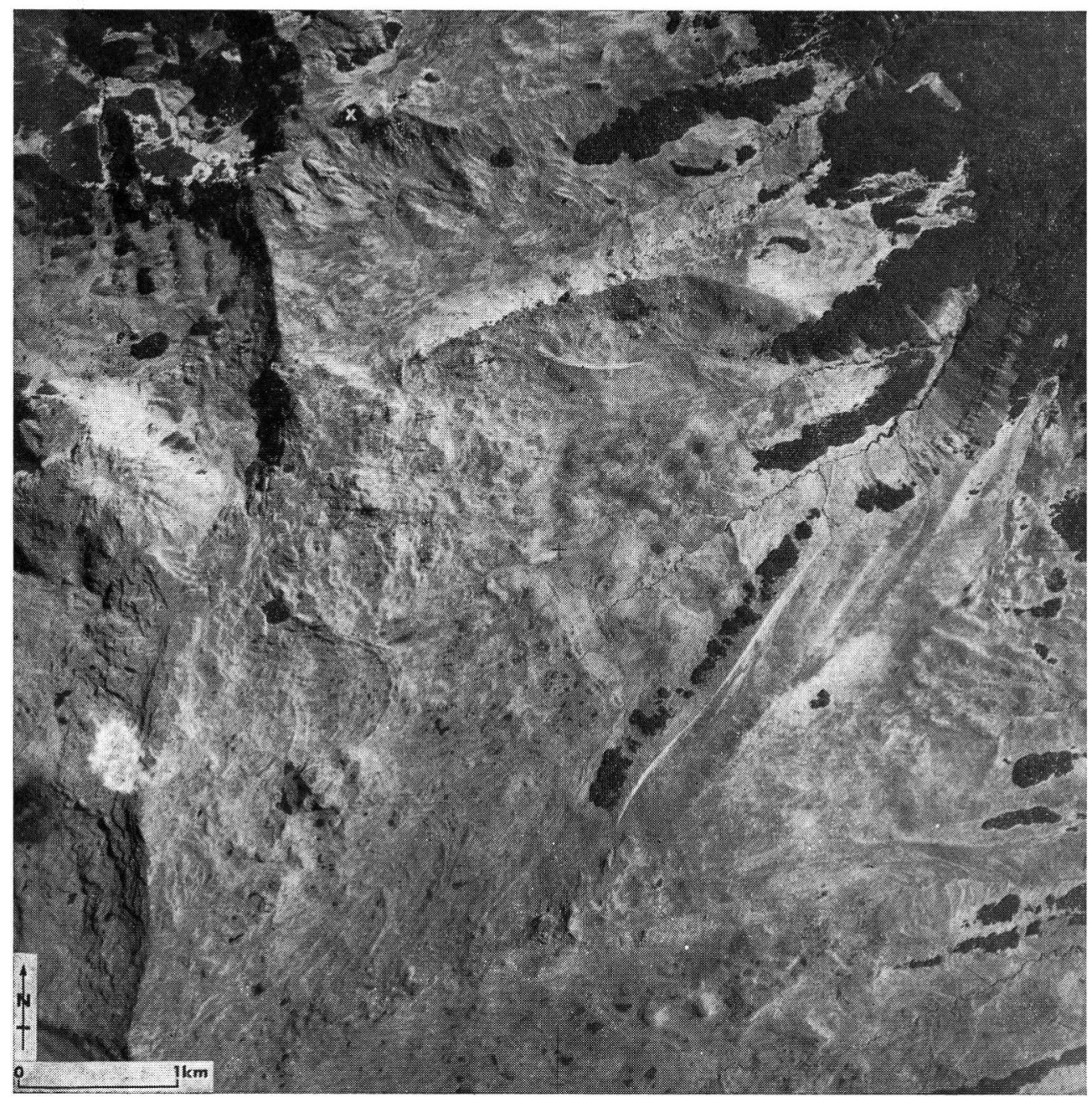

Taf. II: Mt Giluwe, ein erloschener Vulkan zeigt eine typische Kappenvereisung und die eiszeitlichen Endmoränen umgeben den Vulkan wie ein Gürtel. Besonders auffallend sind hier die zahlreichen niedrigen Rückzugsmoränen, die oft bündelweise auf der hier gezeigten flachen, wenig zerschnittenen Ostabdachung vorkommen. Die hier zu sehenden Laven sind alle zwischen 220000 und 320000 Jahre alt. Die palagonitische Breccie im Gogon Tal ist durch ein weißes Kreuz angezeigt (oben links). 


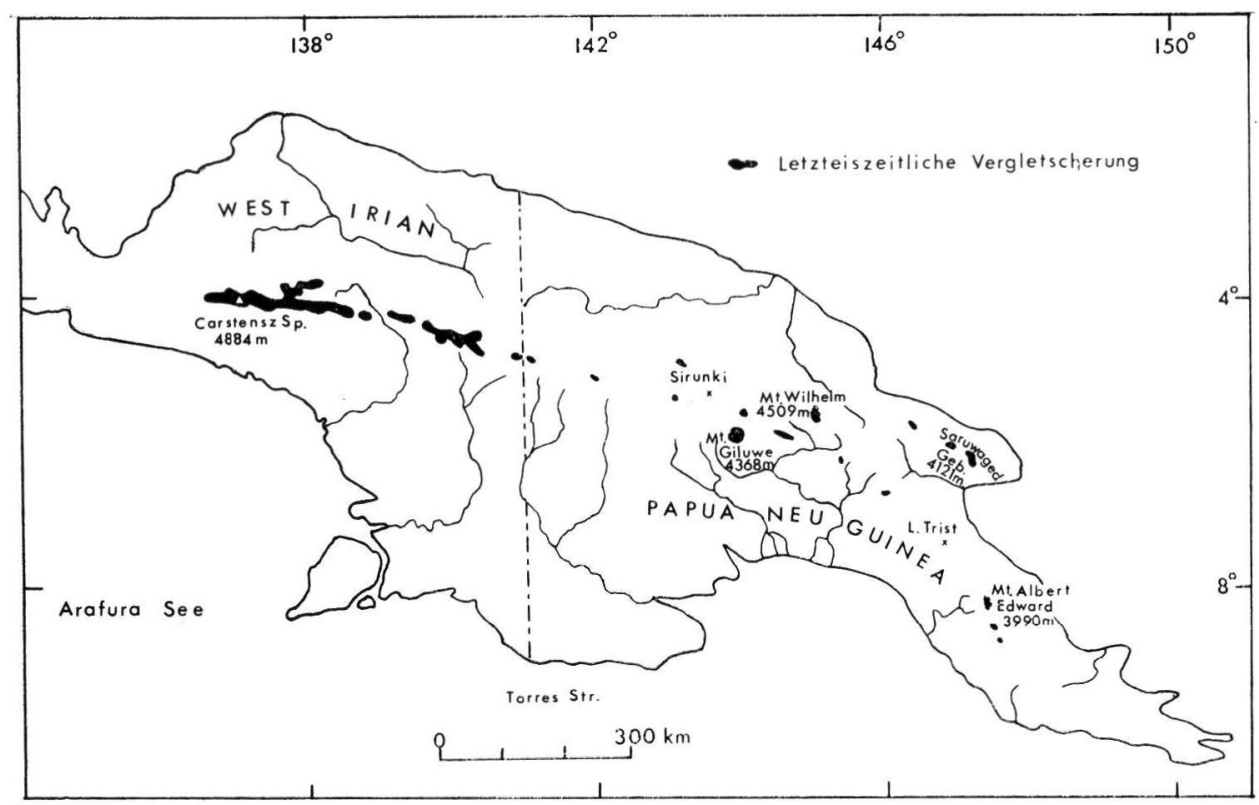

Abb. 1: Übersichtskarte über die Ausdehnung der eiszeitlichen Vergletscherung in Neuguinea.

besaß. ${ }^{14} \mathrm{C}$-Datierungen von Rohtorf in drei Sumpfbecken unmittelbar hinter derartigen Rückzugsmoränen ergaben folgende Ergebnisse. Die zweitälteste Gruppe ist mindestens $13050 \pm 700$ Jahre alt, die zweitjüngste $11250 \pm 550$ Jahre und für ein kleines Becken in unmittelbarer Gipfelnähe wurde ein Alter des Torfs von $9980 \pm 280$ a B.P. ermittelt. Diese Proben wurden von Dr. G. S. Hope und dem Autor im Jahre 1973 gesammelt, und diese neuen Altersangaben ersetzen die in LöfFler (1972) angeführten. Diese Altersdatierungen zeigen, daß um 13000 a B.P. das Eis bereits stark zusammengeschmolzen sein muß, obwohl von der Ausdehnung her die Gletscherkappe fast noch die gleiche Größe aufwies wie zur Maximalausdehnung. Bei 11000 a B.P. war der Kappengletscher sowohl in Mächtigkeit als auch Ausdehnung bereits stark geschwunden und bei rund 10000 B.P. war das Gebirge praktisch eisfrei.

Die Schneegrenze während der Maximalausdehnung lag bei etwa $3550 \mathrm{~m}$ (LöffLER 1972).

\subsection{Mt. Wilhe $1 \mathrm{~m}$}

Im Gegensatz zum Mt. Giluwe war der Vergletscherungstyp am Mt. Wilhelm typisch alpin im Charakter. Das Gebirgsmassiv, das 4509 m Gipfelhöhe erreicht, ist von tief eingesenkten Trogtälern und Karschlüssen zergliedert (Taf. I). In den Karen liegen zahlreiche, malerische Karseen und am Ausgang der Trogtäler mächtige, hohe Moränen, die die Talböden oft un $100 \mathrm{~m}$ übersteigen. Die Kare weisen eine gewisse bevorzugte Exposition auf und zwar sind die meisten nach W ausgerichtet (Taf. I). Diese Asymmetrie wurde von REINER (1960) als das Ergebnis einer präglazialen Asymmetrie des Hauptgrates erklärt. Das Studium von Luftbildern zeigt jedoch, daß diese Asymmetrie nicht nur auf den Hauptgrat beschränkt ist, sondern an allen Seitenspornen und Nebengraten vorhanden 
ist. Die Asymmetrie hat gewisse Ahnlichkeiten mit der in subtropischen Hochgebirgen beobachteten Hangasymmetrie zwischen nord- und südexponierten Hängen (KLAER 1962; Spreitzer 1960). Eine mögliche Erklärung wäre die bereits von Troll (1941) herausgestellte Asymmetrie in der Bestrahlung von ost- und westexponierten Hängen in den Tropen wegen der unterschiedlichen Bewölkungsverhältnisse im Verlauf eines Tages. Die ostexponierten Hänge erhalten im Durchschnitt eine etwas höhere Einstrahlung als die westexponierten, da die frühen Morgenstunden und Vormittage in der Regel wolkenfrei sind, während die Mittagszeit und vor allem die Nachmittage fast immer wolkenverhangen sind und so die Einstrahlung reduzieren. Der Ansatz der Gletscherbildung dürfte daher in Westexposition günstiger sein. Ein Problem bei dieser einleuchtenden Erklärung ist allerdings die Tatsache, daß auf den anderen Hochgebirgen Neuguineas diese Asymmetrie nicht in dieser deutlichen Form ausgebildet ist.

Der Gletscherrückzug am Mt. Wilhelm war ähnlich wie am Mt. Giluwe, allerdings vermissen wir hier die zahlreichen Rückzugsmoränen. Im breiten Pindeaunde-Tal sind lediglich zwei ausgeprägte Rückzugsmoränen zu finden. Der Unterschied in der Anzahl der Rückzugsmoränen reflektiert wahrscheinlich den unterschiedlichen Vergletscherungstyp (Taf. II). Die mächtigen tief eingesenkten Talgletscher des Mt. Wilhelm-Gebiets waren offenbar weniger empfindlich gegen kleinere Klimaschwankungen als die offene, relativ dünne Gletscherkappe auf dem Mt. Giluwe. Der Rückzug am Mt. Wilhelm war daher einheitlicher und zeigt lediglich die Hauptklimaänderung zu wärmeren Temperaturen an.

Die ${ }^{14} \mathrm{C}$-Daten für den Gletscherrückzug am Mt. Wilhelm decken sich weitgehend mit den Ergebnissen am Mt. Giluwe. Nach Hope (1976), der am Mt. Wilhelm vor allem pollenanalytisch arbeitete, begann der Rückzug des Talgletschers aus dem Pindeaunde-Tal um rund 14000 a B.P. Bei etwa 11000 a B.P. war das Eis bis auf ein kleines Gebiet um den Hauptgipfel verschwunden und bei spätestens 9000 a B.P. war das gesamte Gebirge eisfrei. Die pollenanalytischen Ergebnisse werden später diskutiert.

\subsection{Mt. Albert Edward}

Das Mt. Albert Edward-Gebirge liegt im äußersten E Neuguineas und etwa $2^{\circ}$ weiter im $S$ als die anderen Gebirge (Abb. 1). Das Gebirge ist ein Teil der Owen Stanley-Ketten und stellt eine plateauartige Altlandschaft dar, die wahrscheinlich postmiozänen Alters ist. Trotz bescheidener Gipfelhöhe von 3990 m weist das Gebirge ausgedehnte und eindrucksvolle Spuren eiszeitlicher Vereisung auf. Besonders auffallend sind die zahlreichen Kare und Karseen entlang des steilen Gipfelgrats, der das Plateau um rund $300 \mathrm{~m}$ überragt. Der Gipfelgrat weist einen deutlich asymmetrischen Aufbau auf, ähnlich wie der Mt. Wilhelm mit steilen westexponierten Karrücken und weniger steil einfallenden ostexponierten Hängen. Diese Asymmetrie ist jedoch eindeutig gesteinsbedingt und zwar neigt sich die Schieferung des hier anstehenden Phyllits mit rund $30-40^{\circ}$ nach NE und dementsprechend sind die Hänge in Ostauslage weniger steil als die Hänge in Westexposition. Der Hauptkamm stellt praktisch eine Schichtrippe dar. Es ist aus diesem Grund schwierig, hier den Einfluß einer klimatisch bedingten Asymmetrie festzustellen.

Das Plateau war von einem zusammenhängenden Plateaugletscher bedeckt, der ungefähr $90 \mathrm{~km}^{2}$ einnahm. Die Schneegrenze lag mit $3650 \mathrm{~m}$ etwas höher als am Mt. Giluwe und Mt. Wilhelm, wahrscheinlich aufgrund der etwas geringeren Niederschläge und der etwas ausgeprägteren Trockenzeit in diesem Gebiet.

Eine ${ }^{14} \mathrm{C}$-Datierung von Rohtorf in einem Zungenbecken am südlichen Rand des Vergletscherungsgebiets ergab ein Mindestalter von 12000 a B.P. für den Gletscherrückzug (Dr. G. S. Hope frdl. mündl. Mitt.). 


\subsection{Das Saruwaged-Gebirge}

Das Saruwaged-Gebirge ist der höchste Abschnitt des nördlichen Küstengebirges und das einzige nennenswerte Gebiet im Küstengebirge, welches eine eiszeitliche Vergletscherung aufwies (Abb. 2). Der Gipfelbereich ist wie im Mt. Albert Edward-Gebirge plateauartig, allerdings hier bedingt durch mächtige nahezu horizontal lagernde Kalksteinbänke. Der glaziale Formenschatz ist hier nicht sehr eindrucksvoll ausgeprägt trotz einer beachtlichen Gletscherausdehnung von $80 \mathrm{~km}^{2}$. Der Grund hierfür dürfte der flachlagernde Kalkstein sein, der auf Grund seiner Einheitlichkeit wenig Ansätze zur selektiven Glazialerosion bot. Der Vergletscherungstyp war dem des Mt. Albert Edward ähnlich. Ein zusammenhängender Plateaugletscher überdeckte das gesamte Gipfelplateau, und einzelne kurze Talgletscher stießen in die seitlichen Täler vor. Im S und SW, wo das Plateau mit einer $500-800 \mathrm{~m}$ hohen Stufe abbricht, wurden derartige Talgletscher wahrscheinlich von Eislawinen ernährt.

Trotz der küstennahen Lage und der relativ hohen Niederschläge lag die eiszeitliche Schneegrenze im Saruwaged-Gebirge relativ hoch, und zwar mit $3700 \mathrm{~m}$ rund $150 \mathrm{~m}$ höher als am Mt. Giluwe und Mt. Wilhelm. Es ist höchst unwahrscheinlich, daß dieser beachtliche Unterschied einen Temperaturunterschied widerspiegelt, und ein geringerer Niederschlag ist noch unwahrscheinlicher, da die heutigen Niederschlagsverhältnisse eher für eine niedrigere Schneegrenze im Saruwaged-Gebirge sprechen würden. Ich nehme daher an, $\mathrm{daß}$ die relativ hohe Schneegrenze hier auf postglaziale Hebung des Gebirges zurïckzuführen ist (LÖFfLER 1971). Für eine derartige Hebung gibt es an der Küste eindrucksvolle Beweise in der Form von gehobenen Küstenterrassen (CHAppell 1974).

\subsection{Das Carstensz-Gebirge ( $M t$. Jaya)}

Das Carstensz-Gebirge ist mit $4884 \mathrm{~m}$ Gipfelhöhe in der Carstensz-Spitze (nicht $5030 \mathrm{~m}$ wie auf vielen Atlanten angegeben) das höchste Gebirge Neuguineas und das Gebirge mit der ausgedehntesten eiszeitlichen Vergletscherung, die sich über nahezu $1000 \mathrm{~km}^{2}$ erstreckte. Auch hier sind die Moränen der letzten Vereisung ausgezeichnet erhalten, besonders auf einem nördlich des Hauptgrats liegenden Plateau, dem Kemabu-Plateau, auf dem prächtige Moränenrücken mit bis $5 \mathrm{~km}$ Länge und $150 \mathrm{~m}$ Höhe abgelagert wurden (Hope et al. 1976). Diese Moränen liegen in etwa $3400 \mathrm{~m}$ Höhe. Nach S stießen die eiszeitlichen Gletscher in wesentlich tiefere Höhenlagen vor, offensichtlich wegen der unterschiedlichen topographischen Voraussetzungen, denn der relativ flachen, zum Kemabu Plateau hin auslaufenden Nordabdachung steht eine sehr steile Südabdachung gegenüber, und die tiefsten Moränen sind hier in $2300 \mathrm{~m}$ Höhe gefunden worden (Dozy 1938; Hope et al. 1976).

Die Schneegrenze lag hier in etwa 3600-3700 m Höhe. Es wird angenommen, daß diese im Vergleich zu den weiter im E liegenden Gebirgen relativ hohe Schneegrenze einen relativ geringeren Niederschlag im Carstensz-Gebirge reflektiert, der durch das Verlanden der Torres Strait und Arafura-See und dem zeitweiligen Aussetzen der feuchtigkeitsbringenden Südostpassate hervorgerufen wurde.

Der Gletscherrückzug im Carstensz-Gebirge setzte um etwa 14000 a B.P. ein, zwei kleinere Vorstöße konnten für die Zeit um 12500 a B.P. und 11000 a B.P. nachgewiesen werden, und sie werden als das Ergebnis eines deutlich verstärkten Niederschlags aufgrund der einsetzenden Uberflutung der Torres Strait und Arafura-See interpretiert (Hope et al. 1976). Zu welcher Zeit der Gletscherrückzug beendet war, ist nicht bekannt, aber man geht wohl nicht fehl, ihn wie auf den anderen Gebirgen auf etwa 9000-10000 a B.P. festzusetzen. 
Während auf allen bisher besprochenen Gebirgen keine Gletschervorstöße nach 9000 a B.P. stattfanden, konnten für das Carstensz-Gebirge mehrere Vorstöße ab etwa 3000 a B.P. nachgewiesen werden (Hope et al. 1976). Im Yellow Valley, dessen Talschluß vom Carstensz-Gletscher eingenommen wird, konnten in einem Aufschluß 4 übereinanderlagernde, durch organisches Material getrennte Moränen festgestellt werden. ${ }^{14} \mathrm{C}$-Datierungen ergaben folgende Alter für die Moränen. Die älteste Moräne wurde um 3000 a B.P. abgelagert oder etwas vorher, die zweitälteste entstand um etwa 2400 a B.P., die dritte um $1800-1600$ a B.P. und die jüngste wurde nach 1300 a B.P. gebildet (HopE et al. 1976).

\section{Prä-Würm-Vergletscherungen}

Nachweise für ältere Vereisungen sind in Gebieten, in denen die Gletscher nur im Gebirge selbst entwickelt waren und nicht auf ein Vorland vorstießen, immer schwierig zu finden, und Neuguinea ist keine Ausnahme. Ob der Grund hierfür in der starken Abtragung, an der möglicherweise geringeren Ausdehnung älterer Vereisungen in diesen Gebirgen oder aber eventuell an jungen Hebungen liegt, sei im Augenblick dahingestellt.

Auf Neuguinea gibt es zwei Gebiete, in denen Vergletscherungsspuren gefunden wurden, die eindeutig älter sind als die letzte Vereisung. Im Carstensz-Gebirge wurde eindeutiges Moränenmaterial außerhalb der mächtigen letzteiszeitlichen Moränen auf dem Kemabu-Plateau gefunden. Datierungen sind bisher nicht bekannt, aber der fortgeschrittene Verwitterungszustand, im Vergleich zu den praktisch unverwitterten Moränen der letzten Vereisung, läßt auf einen beachtlichen Zeitunterschied schließen (Dow 1968; Hope et al. 1976).

Der andere Nachweis einer älteren Vereisung stammt vom Mt. Giluwe. Der domartige Stratovulkan wird aus einer Serie relativ dünner Lavaströme aufgebaut. Zwischenlagernd mit den normalen Lavaströmen sind an einigen wenigen Stellen Palagonite und palagonitische Breccien vorhanden, die dann entstehen, wenn flüssige Lava plötzlich stark abgekühlt wird. Eine derart drastische Abkühlung läßt auf überlagerndes Eis oder Wasser schließen. Da ein tiefer See im Gipfelbereich des Vulkans unwahrscheinlich ist, geht man wohl nicht fehl, den Palagonit auf die Gegenwart von Eis während des Ausbruchs der Lava zurückzuführen.

Palagonit wurde an zwei Stellen gefunden, zum einen auf der breiten Nordostflanke im Talschluß des Gogon River, zum anderen am östlichen und Hauptgipfel (Löffler et al. 1980). Kalium-Argon-Datierungen der palagonitischen Breccie im Gogon-Tal ergaben Alter von $301000 \pm 40000$ und $285000 \pm 40000$ Jahren, und die die Breccie überlagernde Lava wurde auf $294000 \pm 40000$ Jahre datiert (LöfFlen et al. 1980). Der Palagonit am Hauptgipfel wurde auf $753000 \pm 60000$ und $885000 \pm 80000$ Jahre datiert, der den Palagonit überlagernde Lavastrom auf $743000 \pm 60000$ und $652000 \pm 55000$ Jahre. Da es sich hierbei um Maximalalter handelt, kann man annehmen, daß die älteste Episode glazialer Aktivität um etwa $700000 \pm 60000$ Jahre stattfand, die nächste um $290000 \pm 40000$. Das jüngere Alter wird durch einen Fund im Tamal-Tal, einem dem Gogon-Tal benachbarten Tal auf der Ostabdachung des Vulkans, untermauert. An einer Geländestufe ist eine ungefähr $3 \mathrm{~m}$ mächtige Grundmoräne aufgeschlossen, die von Lava überlagert und unterlagert wird. Das Alter der Laven wurde auf $319000 \pm 20000$ und $301000 \pm 20000$ Jahre für die Lava im Liegenden und $292000 \pm 20000$ Jahre für die Lava im Hangenden ermittelt (LöFfLER et al. 1980). Die in einer früheren Veröffentlichung für die jüngere Lava angegebene Datierung von $340000 \pm 120000$ und $380000 \pm$ 120000 (LÖFFLER 1976) erwies sich aufgrund neuerer Datierungen als inkorrekt (LöFFLER et al. 1980). Diese neuen Ergebnisse zeigen, daß die Bildung der palagonitischen Breccie 
im Gogon-Tal und die Entstehung der Moräne im Tamal-Tal wahrscheinlich der gleichen Vereisung zuzuordnen sind, und nicht wie früher angenommen, zwei eiszeitliche Phasen repräsentieren.

\section{Pollenanalytische Befunde}

Pollenanalytische Arbeiten auf Neuguinea wurden fast ausschließlich von Mitgliedern der Australian National University (ANU) durchgeführt und haben ganz wesentlich zum Verständnis der quartären Geschichte des Landes beigetragen. Die meisten Arbeiten wurden im Hochland durchgeführt, da dort geeignete Stellen für kontinuierliche Pollenablagerung wie Sumpf- und Seebecken in relativ großer Zahl vorhanden sind. Auch im tropischen Tiefland wurden in den letzten Jahren pollenanalytische Arbeiten angesetzt, aber es gelang dort bisher nicht, in Bereiche glazialen Alters vorzudringen, und die Ergebnisse sollen daher hier nicht diskutiert werden. Ich möchte zwei der wichtigsten Arbeiten aus dem Bereich des Hochlands herausgreifen (Walker \& Flenley 1979; Hope 1976), sowie eigene Beobachtungen aus dem Lake Trist-Gebiet mitteilen.

Das bisher vollständigste Pollendiagramm stammt aus einem Seebecken, dem Lake Sirunki, im westlichen Hochland von Ostneuguinea. Das Diagramm reicht praktisch bis an die Grenze der ${ }^{14} \mathrm{C}$-Datierung heran, und zwar bis 32000 a B.P. Das Seebecken liegt in $2500 \mathrm{~m}$ Höhe und ist von steilen Gebirgskämmen umgeben, die rund $3000 \mathrm{~m}$ erreichen. Einzelheiten über die Zusammensetzung und Auswertung des Pollendiagramms sind in der Arbeit von Walker \& Flenley (1979) zu finden. Für die quartäre Entwicklung sind folgende Ergebnisse von Wichtigkeit.

Die Zeit vor etwa 25000 a B.P. war im Sirunki-Gebiet durch das Vorhandensein von oberem Bergwald mit Nothofagusbeständen gekennzeichnet. Um 25000 a B.P. ist eine erste deutliche Anderung im Pollendiagramm festzustellen, und zwar nehmen die Baumpollen deutlich ab und die Pollen subalpiner Pflanzen zu. Ab 21500 a B.P. ist eine Dominanz subalpiner Graspollen zu verzeichnen. Ein leichtes Ansteigen der Temperaturen zwischen 20000 und 18500 a B.P. wird durch eine schwache Zunahme an Baumpollen angezeigt. Zwischen 18500 und 16000 a B.P. herrschen im Siruki-Becken offenbar extreme Bedingungen mit alpinem Grasland, ähnlich wie heute in den höchsten Gebirgsabschnitten oberhalb der natürlichen Waldgrenze, die bei 3800 - 3900 m liegt. Dies deutet nach WALKER \& Flenley (1979) auf eine Temperatursenkung von rund $10^{\circ} \mathrm{C}$ hin und eine Depression der Waldgrenze von 1500 m oder mehr. Nach 15000 a B.P. wird das Grasland allmählich wieder durch oberen Bergwald ersetzt, der schließlich um 9000 a B.P. in unteren Bergwald übergeht, der heute noch diese Höhenstufe bildet.

Die Ergebnisse von Hope (1976) aus dem Mt. Wilhelm-Gebiet stimmen im Prinzip mit den Ergebnissen von WALKER \& FLENLEY (1979) überein, allerdings reichen sie nicht so weit zurück. Die von HOPE untersuchten Ablagerungen befinden sich in verschiedenen Höhenstufen im Pindeaunde-Tal, einem der Haupttäler des Mt. Wilhelm. Im nicht vergletscherten Bereich in $2740 \mathrm{~m}$ Höhe zeigt das Pollenspektrum subalpines Grasland für die Zeit zwischen 22000 und 15500 a B.P. an. Nach 15500 a B.P. ist eine klare Zunahme an Baumpollen festzustellen, und um 10000 a B.P. sind offenbar Verhältnisse erreicht, die den heutigen entsprechen, d. h. die Vegetation wird von unterem Bergwald bestimmt. Im glazialen Bereich, in $3550 \mathrm{~m}$ Höhe beginnt die Akkumulation von organischem Material um etwa 13000 a B.P., der Gletscher hatte sich also um diese Zeit bereits um einige $\mathrm{km}$ von seinem Maximalstand bei $3200 \mathrm{~m}$ zurückgezogen. Dem sich zunächst entwickelnden alpinen und subalpinen Grasland folgt um 10800 a B.P. eine Formation, die durch ein Zunehmen von Büschen und Sträuchern gekennzeichnet ist, bis dann um 9000 a B.P. dichter, von Koniferen dominierter Bergwald vorherrscht. 
In 3910 m Höhe setzt die Rohtorfbildung in einem kleinen glazialen Becken um etwa 11000 a B.P. ein, d. h. das Eis war bereits bis auf einen kleinen Restbestand in unmittelbarer Gipfelnähe verschwunden. Das sich entwickelnde alpine Grasland wird erstaunlicherweise um 8500 a B.P. von subalpinem Wald abgelöst, der dann um 5000 a B.P. wieder verschwindet und durch das heute diese Höhenstufe beherrschende Tussockgrasland ersetzt wird. Hope (1976) nimmt an, daß es sich hierbei um eine kleine Klimaänderung handelt, und zwar um eine Temperatursenkung um $1-2^{\circ} \mathrm{C}$.

Ein etwas anderes Bild zeigen die Pollenuntersuchungen im Lake Trist-Gebiet. Es handelt sich hier nur um vergleichsweise grobe Ergebnisse einer vorläufigen Untersuchung, die leider wegen der ablehnenden Haltung der dortigen Bevölkerungsgruppen nicht weiter verfolgt werden konnte. Dennoch erscheinen mir die Ergebnisse von Wichtigkeit, besonders im Hinblick auf die von Walker \& Flenley (1979) postulierte ungewöhnlich starke Absenkung der Waldgrenze.

Mein Interesse am Lake Trist-Gebiet war zunächst auf die Untersuchung von Karsterscheinungen im ultrabasischen Gestein ausgerichtet (LöfFLER 1979 b), aber das Studium eines kleinen Seebeckens, das eine unterirdische Karstentwässerung aufweist und daher zeitweise trocken liegt, führte mich zu einem interessanten Aufschluß der Seesedimente.

Diese Sedimente sind durch einen kleinen Bach, der den Seeboden durchquert, zerschnitten, so daß am westlichen Rand des Beckens, in unmittelbarer Nähe des unterirdischen Abflusses, die Sedimente fast in ihrer ganzen Mächtigkeit aufgeschlossen sind. Sie bestehen an der Basis aus einer rund 0,6-0,7 m mächtigen Schicht aus organischem Mate-

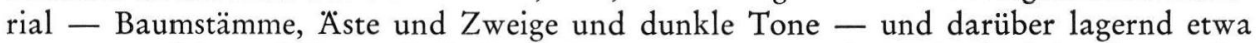
0,9-1,0 $\mathrm{m}$ fein geschichtete, helle Tone. Die organische Schicht ist durch einige dünne, diskontinuierliche Tonschichten untergliedert. ${ }^{14} \mathrm{C}$-Datierungen von Holzfragmenten aus dieser Basisschicht ergaben (von unten nach oben) Alter von $31800 \pm 4000 / 2500,8990 \pm$ $215,37000 \pm 5500 / 3300$ und $>32000$ Jahre vor heute. Die erstaunliche Diskrepanz im Alter zwischen der zweiten Probe und den übrigen ist stratigraphisch nicht zu erklären und ist möglicherweise auf das Vorhandensein von jüngerem, durch den Bach zugeführtem Material in der entnommenen Probe zurückzuführen. Ich nehme an, daß die älteren Angaben das ungefähre Alter der Basisschicht von rund 32000-37000 Jahren vor heute anzeigen.

Die Pollenanalyse der Ablagerungen ergab folgendes: Die Proben aus den organischen Ablagerungen weisen eine hohe Anzahl von Nothofagus-Pollen auf, und zwar stellen diese rund 90\% der Baumpollen dar. Die über den organischen Schichten lagernden Sedimente sind dagegen etwas ärmer an Nothofagus-Pollen, dagegen sind Pollen aquatischer Pflanzen stärker vertreten. Nothofagus ist jedoch weiterhin vorhanden und es besteht kein Zweifel daran, daß während der gesamten Existenz des Beckens der Wald um das Becken ein von Notbofagus dominierter Bergwald gewesen sein muß. Die Gegenwart von Pollen aquatischer Pflanzen deutet lediglich auf eine Veränderung der Vegetation im Seebecken selbst hin, nicht auf eine Veränderung der Vegetation in der Umgebung des Beckens. Die gesamte im Pollenspektrum vorhandene Vegetation ist auch heute noch in der Umgebung des Seebeckens vorhanden, und dies kann als ungefährer Maßstab für den Grad des Klimawechsels in diesem Bereich angesehen werden. Die Waldgrenze ist mit Sicherheit in den letzten 32000 Jahren nicht bis in diesen Höhenbereich vorgedrungen.

Der Übergang vom trockenen, waldbestandenen Becken zum fast ganzjährig wassererfüllten, waldlosen Becken wird durch die deutliche Änderung in der Sedimentation von organischen $\mathrm{zu}$ hauptsächlich nicht organischen Ablagerungen angezeigt. Ob dieses Ereignis auf klimatische Einflüsse (weniger Zufluß) oder auf eine veränderte Karstentwässerung (mehr unterirdischer Abfluß) zurückzuführen ist, kann nicht entschieden werden. Die 
Gegenwart von Nothofagus-Bergwald spricht für relativ hohe (mindestens $2000 \mathrm{~mm}$ ) und gleichmäßig über das Jahr verteilte Niederschläge, und ich möchte daher eher eine veränderte Karstentwässerung für die Trockenhaltung des Beckens verantwortlich machen.

Auch wenn diese Beobachtungen keine feinere Analyse der Vegetationsgeschichte zulassen, so zeigen sie doch die wichtige Tatsache, daß hier in dem kritischen Höhenbereich um $2000 \mathrm{~m}$ keine radikale Veränderung der Vegetation, wie etwa in dem $500 \mathrm{~m}$ höheren Sirunki-Becken, stattfand. Die von Walker \& Flenley (1979) postulierte Temperaturdepression von $10^{\circ} \mathrm{C}$ und Depression der Waldgrenze um $1500 \mathrm{~m}$ und mehr hätte sich doch auch hier in der Gegenwart von Pollen subalpiner oder alpiner Pflanzen zeigen müssen.

\section{Diskussion}

Die glaziale Geschichte Neuguineas reicht möglicherweise bis 700000 Jahre zurück, als am Mt. Giluwe eine Eruption von Lava unter einer Eisdecke stattfand. Das Alter ist jedoch mit einem großen Unsicherheitsfaktor verbunden. Eine weitere Episode vulkanischer Aktivität und Gletscherbildung folgte um etwa 290000-300000. Für dieses Ereignis gibt es zwei unabhängige Nachweise, und es dürfte daher als gesichert angesehen werden. Das Alter von 290000-300 000 Jahren ist etwa synchron mit einer Vereisung auf Hawaii (Porter et al. 1977), auf dem Kilimandscharo (DownIE \& Wilkinson 1972) und stimmt auch ungefähr mit der Klimakurve von Emiliani (1970) und Shackleton \& Opdyke (1973) überein.

Über mögliche Vereisungen zwischen dieser und der letzten haben wir bisher keine Nachweise, aber möglicherweise gehören die Funde am Mt. Carstensz zu einer derartigen Vergletscherung.

Die letzte Vereisung führte auf allen Gebirgen, die rund $3600 \mathrm{~m}$ Höhe übersteigen, zur Gletscherbildung. Der Beginn der Vereisung ist nicht genau bekannt, möglicherweise zeigt der Wechsel von Wald zu Grasland im Sirunki-Becken eine erste Gletscherentwicklung im Gebirge an. Die Maximalausdehnung der Gletscher fand zwischen 18500 und 16000 a B.P. statt; dies ist die Zeit der stärksten Herabsetzung der Vegetationsstufen im Sirunki-Becken. Diese Zeit stimmt auch überein mit der Zeit maximaler Aridität und Dünenbildung in Australien (Bowler et al. 1976). Ab $15000-14000$ a B.P. setzt in Neuguinea der Gletscherrückzug ein, bei 1300 a B.P. waren die Zungenbecken aller untersuchter Gletschergebiete bereits eisfrei und organisches Material wurde dort abgelagert. Der Anstieg der Temperaturen kommt deutlich im Pollenbild zum Ausdruck, sowohl im Sirunki-Becken als auch am Mt. Wilhelm. Der Gletscherrückzug wurde von einzelnen Vorstößen unterbrochen, die jedoch lediglich im Mt. Giluwe-Gebirge zur Ablagerung von zahlreichen Rückzugsmoränen führte. Dies lag wahrscheinlich an der größeren Empfindlichkeit des offenen Kappengletschers gegenüber kleineren Klimaschwankungen. Im Carstensz-Gebirge wurden zwei kleinere Vorstöße für die Zeit von 12500 und 11000 a B.P. festgestellt, und diese reflektieren möglicherweise eine Niederschlagszunahme in diesem Gebiet, verursacht durch die Überflutung der Torres Strait und Arafura-See.

Um 10000-9000 a B.P. waren die Gletscher Neuguineas praktisch verschwunden und zwar wahrscheinlich selbst dort, wo wir heute wieder kleinere Gletscher vorfinden. Dies wird aus den pollenanalytischen Untersuchungen von Hope (1976) geschlossen, die anzeigen, daß die Zeit zwischen 9000 und 5000 a B.P. etwas wärmer war als heute. Die heutigen Gletscher bildeten sich wahrscheinlich irgendwann zwischen 5000 und 3000 a B.P., denn für die Zeit um oder vor 3000 a B.P. konnte ein deutlicher Gletschervorstoß nachgewiesen werden, dem drei weitere um $2400,1800-1600$ und nach 1300 a B.P. folgten. 
Die pleistozäne Schneegrenze lag bei durchschnittlich $3550 \mathrm{~m}$, aber es gab einige Schwankungen aufgrund klimatischer und tektonischer Bedingungen. Die heutige Schneegrenze kann auf etwa 4600 m angesetzt werden, und die eiszeitliche Schneegrenzdepression liegt damit bei rund $1000 \mathrm{~m}$.

Die Rekonstruktion des eiszeitlichen Klimas auf Neuguinea ist mit einigen Schwierigkeiten verbunden, da glazialmorphologische und pollenanalytische Befunde unterschiedliche Depressionen der Höhenstufen anzeigen. Die Schneegrenzdepression von $1000 \mathrm{~m}$ würde einer Temperaturdepression von rund $5,5^{\circ} \mathrm{C}$ entsprechen, nimmt man den heutigen Temperaturgradienten als Grundlage. WALKeR \& Flenley (1979) postulieren jedoch eine Temperaturdepression von rund $10^{\circ} \mathrm{C}$.

Diese erstaunliche Absenkung, die ja zu einer starken Kompression der tropischen Vegetationshöhenstufen hätte führen müssen, ist jedoch aus verschiedenen Gründen schwer verständlich. Erstens konnte im Lake Trist-Gebiet keinerlei Hinweis für eine derartig drastische Klimaänderung, bzw. Wechsel im Vegetationsbild in dem kritischen Höhenbereich von $2000 \mathrm{~m}$ gefunden werden. Eine derartige Temperaturdepression hätte zumindest eine deutliche Änderung in der Zusammensetzung des Waldes zeigen müssen, der das Karstbecken umgibt. Zweitens, um eine Depression der Grenze Wald/Grasland um mindestens das eineinhalbfache der Schneegrenzdepression zu erreichen, müßten beachtliche klimatische Veränderungen stattfinden, und zwar zusätzlich zur Temperaturdepression. So müßte z. B. der Niederschlag beträchtlich geringer gewesen sein als heute und das Tageszeitenklima, das heute sehr ausgeglichen ist, hätte wesentlich größere tägliche Schwankungen aufweisen müssen, etwa ähnlich wie heute am Mt. Kenia, wo der Höhenabstand zwischen Waldgrenze und Schneegrenze etwa $1500 \mathrm{~m}$ beträgt (HASTENRATH 1973). Die heutige und pleistozäne Schneegrenze Neuguineas gehören jedoch zu den niedrigsten Schneegrenzen der tropischen Gebirge und deuten auf hohe Niederschläge hin. Eine deutliche Absenkung des Niederschlags hätte in einer relativ hohen Schneegrenze resultieren müssen.

Eine Verringerung des Niederschlags ist allerdings für den tropischen Bereich Queenslands wie überhaupt für den australischen Kontinent nachgewiesen (BOwLER et al. 1976) und auch für das südliche Tiefland Neuguineas wahrscheinlich (NIx \& Kalma 1972). Die hohen Gebirge Neuguineas erhalten jedoch vor allem orographisch bedingten Niederschlag und liegen außerdem im zentralen Bereich der immerfeuchten Tropen, so daß es unwahrscheinlich ist, daß sie nennenswert von diesen Niederschlagsverhältnissen beeinflußt wurden. Ich möchte daher annehmen, daß die ungewöhnlich tiefe Lage des alpinen Graslands im Sirunki-Becken vor allem lokale Bedingungen widerspiegelt, und zwar hauptsächlich die Beckenlage, die zum einen wesentlich frostempfindlicher ist als Hanglagen, zum anderen auch durch die starke edaphische Feuchte den Wuchs von Wald verhinderte. Ahnliche tiefliegende Becken mit natürlichen Grasformationen sind auch heute weit unterhalb der natürlichen Waldgrenze zu finden (PAIJMAns \& Löffler 1972).

Ein anderes interessantes Phänomen der pleistozänen Temperaturabsenkung auf Neuguinea ist der geringe Effekt, den dies auf andere geomorphologische Prozesse hatte. Zwar resultierte die Herabsenkung der Schneegrenze um 1000 m sicherlich in einem Herabsenken der Solifluktionsgrenze, aber es wurden bisher noch keine nennenswerten Hinweise auf kaltzeitliche Solifluktionserscheinungen außerhalb der Grenzen der eiszeitlichen Vergletscherung gefunden. Diese Tatsache ist jedoch nicht verwunderlich, wenn man sich die heutigen Verhältnisse betrachtet. Echte Solifluktionserscheinungen wie Streifenböden, Wanderschuttdecken und Auffrierböden treten erst ab etwa $4350 \mathrm{~m}$ auf, d. h. sie liegen praktisch in unmittelbarer Nähe der Schneegrenze, die auf $4600 \mathrm{~m}$ Höhe angesetzt werden kann. Der Solifluktionsbereich ist damit auf eine Höhenspanne von nur etwa $250 \mathrm{~m}$ eingeengt, sicherlich das Ergebnis der extremen Einförmigkeit des Tageszeitenklimas 
mit minimalen Temperaturschwankungen und einem schmalen Höhenbereich von allnächtlichen Frösten (LÖFfLER 1975). Während der eiszeitlichen Vergletscherung dehnte sich das Eis auf den meisten Gebirgen jedoch bis in Höhenlagen um $3200 \mathrm{~m}$ aus, d. h. die Eisfront lag meist 300-400 m tiefer als die Schneegrenze und bedeckte damit diejenigen Bereiche, die klimatisch für solifluidale Vorgänge geeignet gewesen wären, nämlich die Gebiete mit regelmäßigen Nachtfrösten.

Das einheitliche tropische Tageszeitenklima begünstigt die Entwicklung von Gletschern auf Plateaus und plateauartigen Erhebungen. Nennenswerte Expositionsunterschiede treten auf derartigen Gebirgen nicht auf. Sind Gebirge jedoch stark zergliedert und zerschnitten wie z. B. der Mt. Wilhelm, der tief eingesenkte Trogtäler aufweist, so ist ein gewisser Unterschied in West- und Ostexposition zu beobachten. Dies drückt sich in einer bevorzugten Gletscherentwicklung in Westauslage und einer daraus resultierenden Hangasymmetrie mit steilen felsigen Westhängen und weniger steilen „glatten “ Osthängen aus. Der Grund hierfür dürfte in der unterschiedlichen Wirkung der Sonneneinstrahlung auf ost- und westexponierte Hänge liegen, hervorgerufen durch die unterschiedlichen Bewölkungsverhältnisse im Verlauf eines Tages.

Ein weiteres Merkmal der tropischen Vergletscherung ist der ausgezeichnete Erhaltungszustand der Moränen und der Mangel an fluvioglazialen Ablagerungen, beides ein Ergebnis des einförmigen Tageszeitenklimas der Tropen und dem über das ganze Jahr verteilten Schmelzregime der Gletscher.

Verf. ist Frau J. Guppy, Dept. Biogeography and Geomorphology, Australian National University für die Pollenanalyse zu Dank verpflichtet. Die ${ }^{14} \mathrm{C}$-Datierungen wurden von Geochron Laboratories, die K-Ar Datierungen von AmDEL (Australian Mineral Development Laboratories) durchgeführt.

\section{Schriftenverzeichnis}

Bowler, J. M. \& Hope, G. S. \& Jennings, J. N. \& Singh, G. \& Walker, D. (1976): Late Quaternary climates of Australia and New Guinea. - Quat. Res., 6: 359-394; New York.

Chappell, J. (1974): Geology of coral terraces, Huon Peninsula, New Guinea. - Bull. Geol. Soc. Am., 85: 553-570; New York.

Detzner, H. (1933): Der Saruwaged und seine östlichen und südöstlichen Anschlußgebirge. Kolon. Rdsch., Jg. 1933: 209-221; Berlin.

Dow, D. B. (1968): A geological reconnaissance in the Nassau Range, West New Guinea. Geol. en Mijnbouw, 47: 37-46; Den Haag.

Downie, C. \& Wilkinson, P. (1972): The geology of Kilimanjaro. - The Department of Geology, The University of Sheffield.

Dozy, J. J. (1938): Eine Gletscherwelt in niederländisch Neuguinea. - Z. Gletk., 26: 45-51; Leipzig.

Emilinni, C. (1970): Pleistocene paleo-temperatures. - Science, 168: 822-825; Washington D.C.

Hastenrath, S. (1973): Observations on the periglacial morphology of Mts Kenia and Kilimandscharo, East Africa. - Z. Geomorph. N.F., Suppl. Bd., 16: 161-179; Berlin, Stuttgart.

Hope, G. S. (1976): The vegetational history of Mt. Wilhelm, Papua New Guinea. - Jour. Ecol., 64: 627-664; Oxford.

- \& Peterson, J. A. (1975): Glaciation and vegetation in the high New Guinea mountains. Bull. Roy. Soc. N.Z., 13: 155-162; Wellington N.Z.

- - \& RadoK, U. \& Allison, U. (1976): The equatorial glaciers of New Guinea. - 244 S.; Rotterdam (A. A. Balkema).

KLAER, W. (1962): Die periglaziale Höhenstufe in den Gebirgen Vorderasiens - Ein Beitrag zur Morphogenese der Hochgebirge in den subtropischen Breiten. - Z. Geomorph., N.F., 6: 17-32; Berlin, Stuttgart. 
Löfrler, E. (1970): Evidence of Pleistocene glaciation in East Papua. - Austr. Geogr. Stud., 8: 16-26; Hobart.

- (1971): The Pleistocene glaciation of the Saruwaged Range, Territory of New Guinea. Aust. Geogr., 11: 463-472; Sydney.

- (1972): Pleistocene glaciation in Papua New Guinea. - Z. Geomorph., N.F. Suppl. Bd., 13: 32-58; Berlin, Stuttgart.

- (1975): Beobachtungen zur periglazialen Höhenstufe in den Hochgebirgen von Papua New Guinea. - Erdkunde, 29: 285-292; Bonn.

- (1976): Potassium - argon dates and pre-Würm glaciations of Mount Giluwe volcano, Papua New Guinea. - Z. Gletk. u. Glazgeol., 12: 55-62; Innsbruck.

- (1979 a): Ursprung und Verbreitung der Paramo-Grasländer in Ostneuguinea. - Erdkunde, 33: 226-236; Bonn.

- (1979 b): Formen der subterranen Materialabfuhr in den feuchten Tropen. - Die Erde, 110: 135-144; Berlin.

- \& Mackenzie, D. \& Webb, A. (1980): Potassium - argon ages of some Papua New Guinea highland volcanoes and their relevance to Pleistocene geomorphic history. - Jour. Geol. Soc. Aust., 27; Adelaide.

Nix, H. A. \& Kalma, J. D. (1972): Climate as a dominant control in the biogeography of northern Australia and New Guinea. - In: WALKER, D. (Hrsg.): Bridge and Barrier: the natural and cultural history of Torres Strait. S. 61-91. (Dept. Biogeogr. and Gcomorph. Publ. BG/3, Aust. Nat. Univ. Press).

Paijmans, K. \& Löffler, E. (1972): High-altitude forests and grasslands of Mt Albert Edward, New Guinea. - J. Trop. Geogr., 34: 58-64; Singapore, Kuala Lumpur.

Porter, S. C. \& Stuiver, M. \& YANG, I. C. (1977): Chronology of Hawaiian glaciations. Science, 195: 61-63; Washington D.C.

Reiner, E. (1960): The glaciation of Mt Wilhelm, Australian New Guinea. - Geogr. Rev., 50: 491-503; New York.

Shackleton, N. J. \& Opdyke, N. D. (1973): Oxygen isotope and paleomagnetic stratigraphy of equatorial Pacific core V 28-238. Oxygen isotope temperatures and ice volume on a 105 year and 106 year scale. - Quat. Res., 3, 39-55; New York.

Spreitzer, H. (1960): Hangformung und Asymmetrie der Bergrücken in den Alpen und im Taurus. - Z. Geomorph., Suppl. Bd. 1: 211-236; Berlin, Stuttgart.

Tricart, J. \& Cailleux, A. \& Raynal, R. (1962): Les particularités de la morphogénèse dans les régions de montagne. - C.D.U., 5 pl. de la Sorbonne, Paris V.

Troll, C. (1941): Studien zur vergleichenden Geographie der Hochgebirge der Erde. - Hauptversammlung Ges. Freunde und Förderer der Rheinischen Friedrich-Wilhelms-Universität. Bonn, Ber. 23: 49-96.

WALKER, D. (1970): The changing vegetation of the montane tropics. - Search, 1: 217-221; Sidney.

- \& Flenley, J. R. (1979): Late Quaternary vegetational history of the Enga Province of upland Papua New Guinea. - Phil. Trans. Roy. Soc. London. Biol. Ser., 286: 265-344; London.

Wollaston, A. F. R. (1914): An expedition to Dutch New Guinea. - Geogr. Journ., 43: 248273; London. 
E. Löffler 